\title{
ACMG statement on access to reproductive options after prenatal diagnosis
}

\author{
ACMG Board of Directors
}

19 July 2013-Bethesda, MD-The American College of Medical Genetics and Genomics (ACMG) is concerned with the enactment of laws in some states that prevent or restrict access to termination of pregnancy after prenatal diagnosis of genetic disorders or congenital anomalies. The practice of medical genetics is predicated on the principle of providing patients with accurate information on the genetic disorder or congenital anomaly that affects them, a member of their family, or an unborn fetus, and then discussing the management options that are available. The ACMG believes strongly that a balanced discussion of options, including termination of pregnancy, should be available to pregnant couples where their fetus has been diagnosed with a genetic disorder or congenital anomaly. This is accomplished on a case-by-case basis via discussions between the patient's care provider, a medical geneticist/genetic counselor, and the pregnant couple, with the goal of serving the medical needs of the couple to choose a safe and personally acceptable management plan. Access to safe and legal termination of pregnancy for genetic disorders or congenital anomalies that may be diagnosed prenatally is a critically important option for some pregnant couples, and the ACMG strongly opposes legislation that places limits on this access.

\section{DISCLOSURE}

The author declares no conflict of interest. 